Article

\title{
Green Citrus Detection and Counting in Orchards Based on YOLOv5-CS and AI Edge System
}

\author{
Shilei Lyu ${ }^{1,2,3,4}$, Ruiyao Li ${ }^{1,3}$, Yawen Zhao ${ }^{1,3}$, Zhen $\mathrm{Li}^{1,2,3,4, *}$, Renjie Fan ${ }^{1,3}$ and Siying Liu ${ }^{1}$ \\ 1 College of Electronic Engineering (College of Artificial Intelligence), South China Agricultural University, \\ Guangzhou 510642, China; lvshilei@scau.edu.cn (S.L.); 20203162097@stu.scau.edu.cn (R.L.); \\ w844718456@163.com (Y.Z.); richard.fan@stu.scau.edu.cn (R.F.); 201934910820@stu.scau.edu.cn (S.L.) \\ 2 Guangdong Laboratory for Lingnan Modern Agriculture, Guangzhou 510642, China \\ 3 Pazhou Lab, Guangzhou 510330, China \\ 4 Division of Citrus Machinery, China Agriculture Research System of MOF and MARA, \\ Guangzhou 510642, China \\ * Correspondence: lizhen@scau.edu.cn; Tel.: +86-1361-018-9829
}

Citation: Lyu, S.; Li, R.; Zhao, Y.; Li, Z.; Fan, R.; Liu, S. Green Citrus

Detection and Counting in Orchards Based on YOLOv5-CS and AI Edge

System. Sensors 2022, 22, 576.

https://doi.org/10.3390/s22020576

Academic Editor: Benoit Vozel

Received: 30 November 2021

Accepted: 10 January 2022

Published: 12 January 2022

Publisher's Note: MDPI stays neutral with regard to jurisdictional claims in published maps and institutional affiliations.

Copyright: (C) 2022 by the authors. Licensee MDPI, Basel, Switzerland. This article is an open access article distributed under the terms and conditions of the Creative Commons Attribution (CC BY) license (https:// creativecommons.org/licenses/by/ $4.0 /)$.

\begin{abstract}
Green citrus detection in citrus orchards provides reliable support for production management chains, such as fruit thinning, sunburn prevention and yield estimation. In this paper, we proposed a lightweight object detection YOLOv5-CS (Citrus Sort) model to realize object detection and the accurate counting of green citrus in the natural environment. First, we employ image rotation codes to improve the generalization ability of the model. Second, in the backbone, a convolutional layer is replaced by a convolutional block attention module, and a detection layer is embedded to improve the detection accuracy of the little citrus. Third, both the loss function CIoU (Complete Intersection over Union) and cosine annealing algorithm are used to get the better training effect of the model. Finally, our model is migrated and deployed to the AI (Artificial Intelligence) edge system. Furthermore, we apply the scene segmentation method using the "virtual region" to achieve accurate counting of the green citrus, thereby forming an embedded system of green citrus counting by edge computing. The results show that the mAP@.5 of the YOLOv5-CS model for green citrus was $98.23 \%$, and the recall is $97.66 \%$. The inference speed of YOLOv5-CS detecting a picture on the server is $0.017 \mathrm{~s}$, and the inference speed on Nvidia Jetson Xavier NX is $0.037 \mathrm{~s}$. The detection and counting frame rate of the AI edge system-side counting system is 28 FPS, which meets the counting requirements of green citrus.
\end{abstract}

Keywords: green citrus; object detection; virtual region; YOLOv5-CS; AI edge system

\section{Introduction}

The collection and monitoring of information throughout the fruit growth cycle essentially play a guiding role in the delicacy management of the fruit industry and has become the critical technical basis for constructing an intelligent orchard [1-3]. Recently, the application of computer vision technology for fruit detection has become a research hotspot. Especially, the detecting, tracking, and counting of green citrus are essential for predicting the yield of orchards during harvest. Research could provide reliable data support for orchard production management such as fruit thinning, sunburn prevention, and unmanned picking $[4,5]$. To realize the intelligent detection of fruits in the natural environment, researchers worldwide have successively explored and studied several solutions. Li et al. proposed an improved YOLOv3 lightweight model combined with the Mobile Net method for ripe fruit detection and applied it to dragon fruit detection in the actual environment [6]. Bi et al. used multiple segmentation methods to recognize citrus targets in the natural environment and improved multi-scale image detection and real-time performance of the citrus object detection model [7]. Xiong et al. applied the multi-scale convolutional neural network, the Des-YOLOv3 model, to realize the detection 
of citrus in the night environment [8]. Li et al. proposed the SSD-ResNet18 improved model to realize the real-time detection and classification of normal citrus, epidermal lesions, and mechanically damaged citrus [9]. Lyu et al. proposed the YOLOv3-LITE lightweight network model to realize real-time detection of mature citrus [10]. The colors of green fruits are similar to those of fruit tree leaves in tropical and subtropical evergreen orchards. Thus, Zheng et al. proposed the YOLO BP network to detect green citrus in the natural environment, and the results showed that the accuracy, recall, mean average precision (mAP), and detection speed of YOLO BP were 86\%, 91\% and 91.55\% and 18 frames per second (FPS), respectively [11]. Kuznetsova et al. used YOLOv3 and YOLOv5 in general and close-up images. The average apple detection time was $19 \mathrm{~ms}$ with FPR at $7.8 \%$ and FNR at 9.2\% using YOLOv3 and with FPR at 3.5\% and FNR at 2.8\% using YOLOv5 [12]. Parico and Ahamed proposed a real-time pear fruit counter using YOLOv4 and DeepSORT, with an AP@0.50 of 98\% [13]. Yan et al. proposed a light-weight fruit target real-time detection method for the apple picking robot based on improved YOLOv5, with an mAP of $86.75 \%$ [14]. Chen et al. proposed an improved YOLOv4 network structure to detect small citrus against a complex background, with an average increase in accuracy of 3.15\% (from 92.89\% to 96.04\%) [15]. Xue et al. applied an improved YOLOv2 object detection model to accurately identify immature mangoes in an orchard environment [16]. Shi et al. also proposed a generalized attribution pruning detection method, which strips the subnetworks from large-scale networks for the real-time detection of mangoes [17]. Mu et al. performed transfer learning using a convolutional neural network, the R-CNN network, and Resnet-101 to detect immature tomatoes [18]. Wang et al. proposed an improved YOLOv5 model to detect small apples using the channel pruning method, with an average detection time of $8 \mathrm{~ms}$ per image. However, it was not applied to the mobile terminal for identification [19].

Presently, the majority of researchers focus on improving the static detection effect of different fruit targets [20-22]. However, related studies on the dynamic tracking and accurate counting of green citrus have seen less attention. Most citrus orchards in China are situated in hills and mountains, and the working environment of the orchards is complicated and changeable. Green citrus detection in orchards in the natural environment, tracking counting, and yield prediction of green citrus targets are more in line with the production needs of citrus orchards in China. In this paper, we adopted software and hardware co-design. We proposed an improved YOLOv5-CS (Citrus Sort) lightweight object detection model and migrated to the AI edge system platform to realize the intelligent detection of green citrus. Besides, using "virtual region", scene segmentation was proposed to count the green citrus accurately. Thus, an embedded system for the intelligent detection of citrus orchards using edge computing was designed and realized.

\section{Related Works}

\subsection{YOLO Models}

Object detection, tracking and counting are vital techniques for realizing citrus fruit quantity statistics. According to the candidate region, object detection can be divided into two: single-stage object detector and two-stage object detector [23]. The single-stage object detector is an object detection model based on regression analysis, which omits the candidate region generation stage and directly obtains object classification and location information. The classic networks include YOLO, SSD, SqueezeDet and DetectNet. The first-level network of the two-stage object detector is used for the candidate region extraction. Candidate regions containing detection targets are selected from the input image, mainly through selective search or edge box model. The second-level network classifies the extracted candidate region and performs precise coordinate regression. The typical networks include a series of R-CNN models. Since the efficiency of the two-stage detection method using the candidate region does not meet real-time requirements and its computational cost is high, we proposed a YOLOv5-based single-stage detection model, theYOLOv5-CS model. In 2015, Redmon et al. proposed the YOLO model [24], which 
divided an image into $S^{*} S$ grids and by the center of the object in the grid on the prediction. However, its recall rate and detection accuracy are relatively low. Figure 1 shows a diagram of the YOLO object detection.
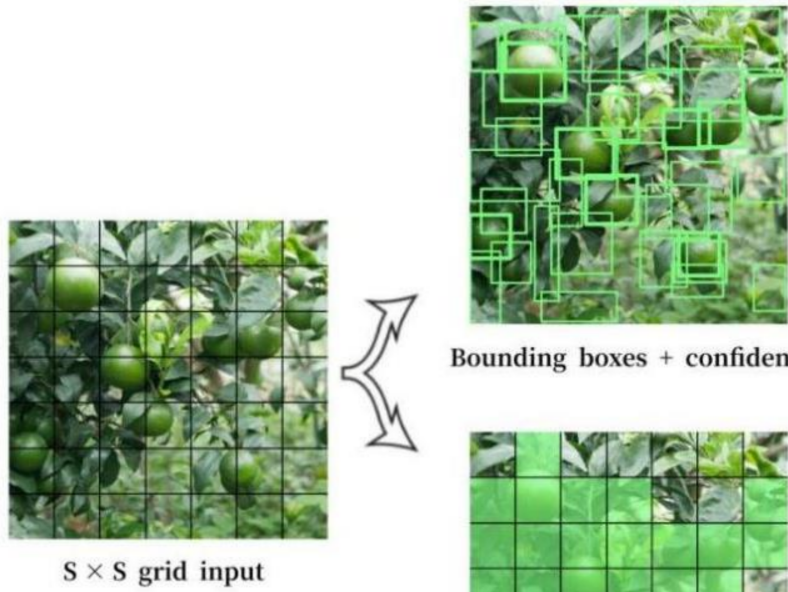

Bounding boxes + confidence

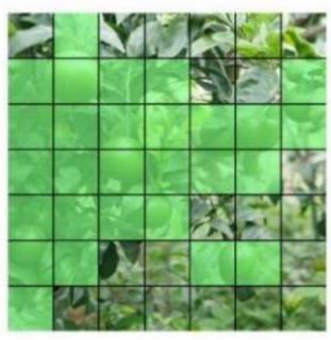

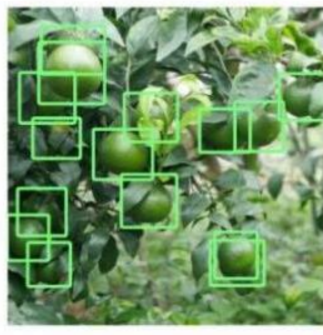

Final detections

Class probability map

Figure 1. YOLO object detection.

In 2017, Redmon et al. proposed the YOLOv2 model [25], which uses the K-means clustering method to automatically select the best initial box, thereby improving the detection effect and speed compared to the previous version. Furthermore, in 2018, they proposed YOLOv3 and used the new Darknet-53 residual network for feature extraction, and three feature maps of different scales to predict [26]. In 2020, Bochkovskiy et al. proposed YOLOv4, which greatly improves the detection accuracy of the model [27]. Jocher proposed the YOLOv5 model in the same year, which is a lightweight network with a size of 27MB [28]. Here, we focus on the YOLOv5 model for detecting green citrus and other related research on quantitative statistics. The YOLOv5 model consists of four parts: the input layer, backbone network, neck network, and output detection layer. Figure 2 shows its network structure diagram.

\subsubsection{Input}

YOLOv5 applied the Mosaic data augmentation method in the input layer. Then, it added a function to adjust the anchor box and the picture adaptively. In each training process, whether to adopt the adaptive adjustment of the anchor box and the image or not can be set for actual purposes. In this paper, we used different lengths and widths of the dataset pictures, so the size of the input pictures in the YOLOv5 network was uniformly modified to $416^{*} 416$, thereby improving the inference and detection speeds.

\subsubsection{Backbone}

The focus module was applied to the backbone network of YOLOv5 to slice the input image. Take the unified image as an example, the image of $416^{*} 416^{*} 3$ was put into the focus module to produce a picture of $208^{*} 208^{*} 12$ after the slicing operation. After that, the image undergoes the convolution operation of 32 convolution kernels, and finally becomes a feature map of $208^{*} 208^{*} 32$. Figure 3 shows the slicing operator of the focus module. 


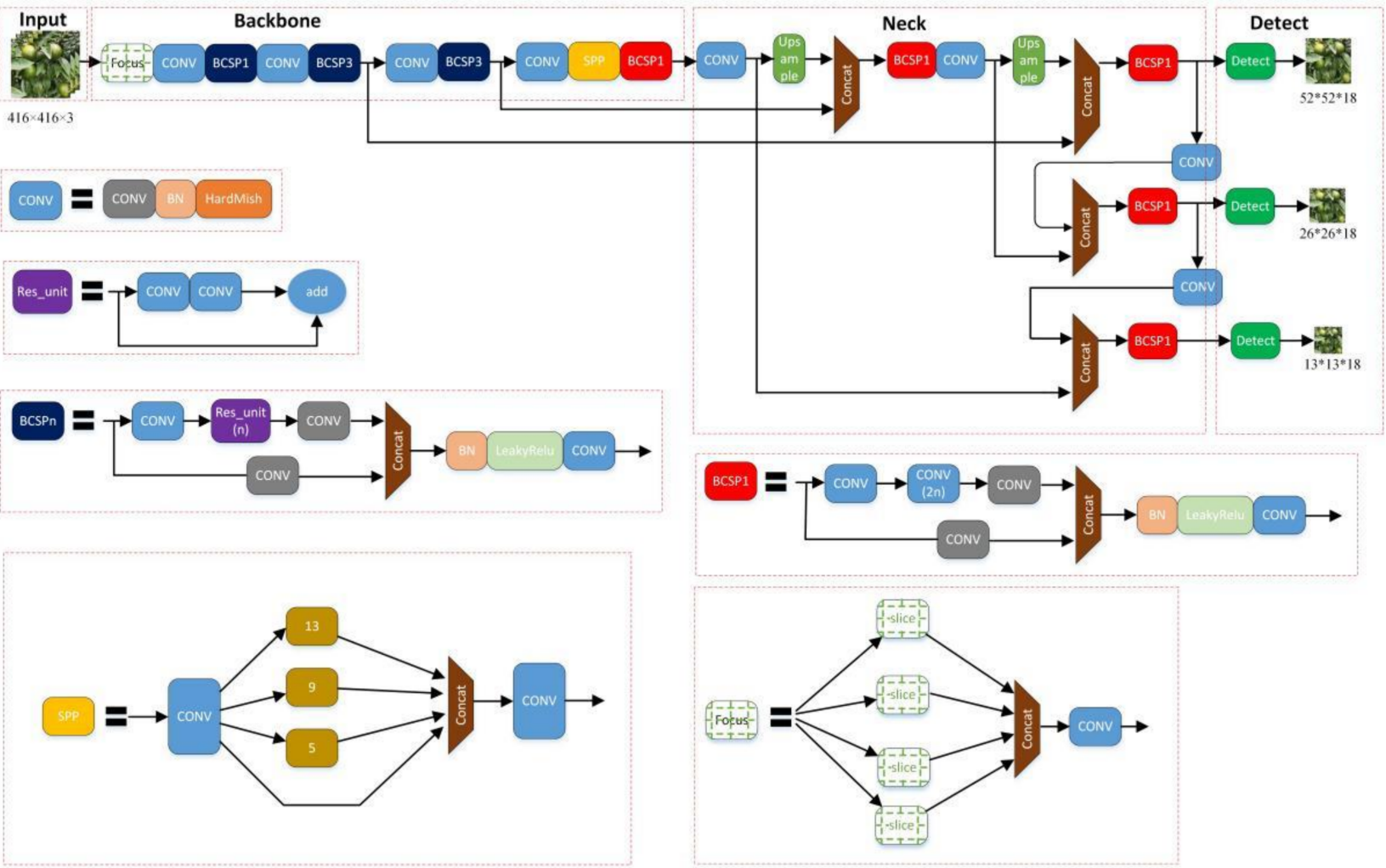

Figure 2. YOLOv5 Architecture.
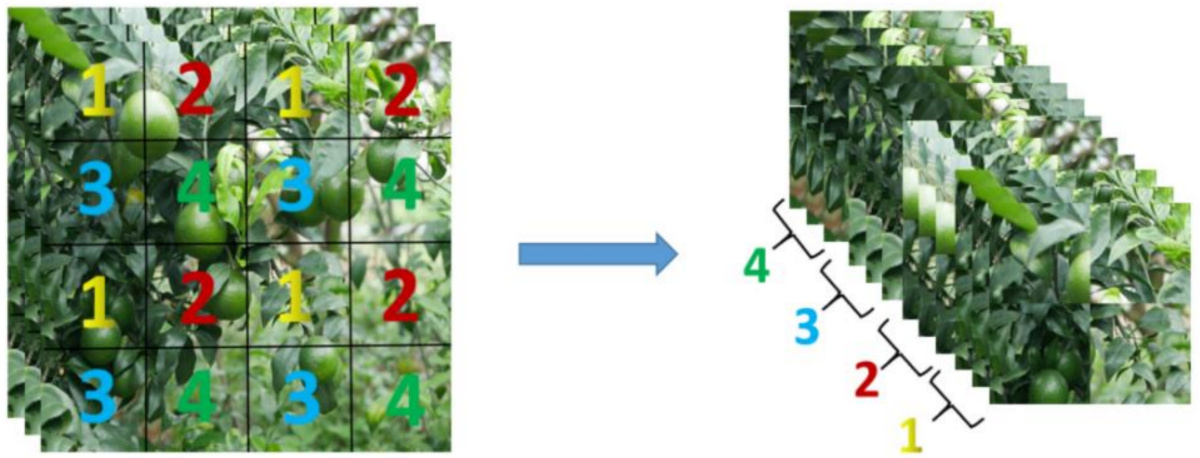

Figure 3. Slicing operation for subimage 1-4.

Additionally, the backbone network also includes the BCSP and SPP modules $[29,30]$. From Figure 2, blue BCSPn represents a module with a residual structure, and red BCSP1 represents a module without a residual structure. The BCSP module is used for improving the learning ability of the convolutional neural network, by making the model smaller while ensuring accuracy. This is conducive for the subsequent migration and deployment of the model on the AI (Artificial Intelligence) edge system. The SPP module is the spatial pyramid pooling module. The three pooling cores are $13^{*} 13,5 * 5$, and $9^{*} 9$, respectively. The last one has no pooling operation but directly joins the Concat module with the other three channels and, finally, passes through Conv layer output. This module is beneficial for increasing the receptive field and the calculation speed does not decrease.

\subsubsection{Neck}

The Yolov5 neck network adopts an FPN structure [31], which is mainly used to generate feature pyramids and enhance the model's ability to detect objects of different scales. Furthermore, it is used to recognize different sizes and standards of the same body. 
Figure 4 shows that after a couple of downsamples, the detected module outputs $52 * 52$, $26^{*} 26,13^{*} 13$ respectively.

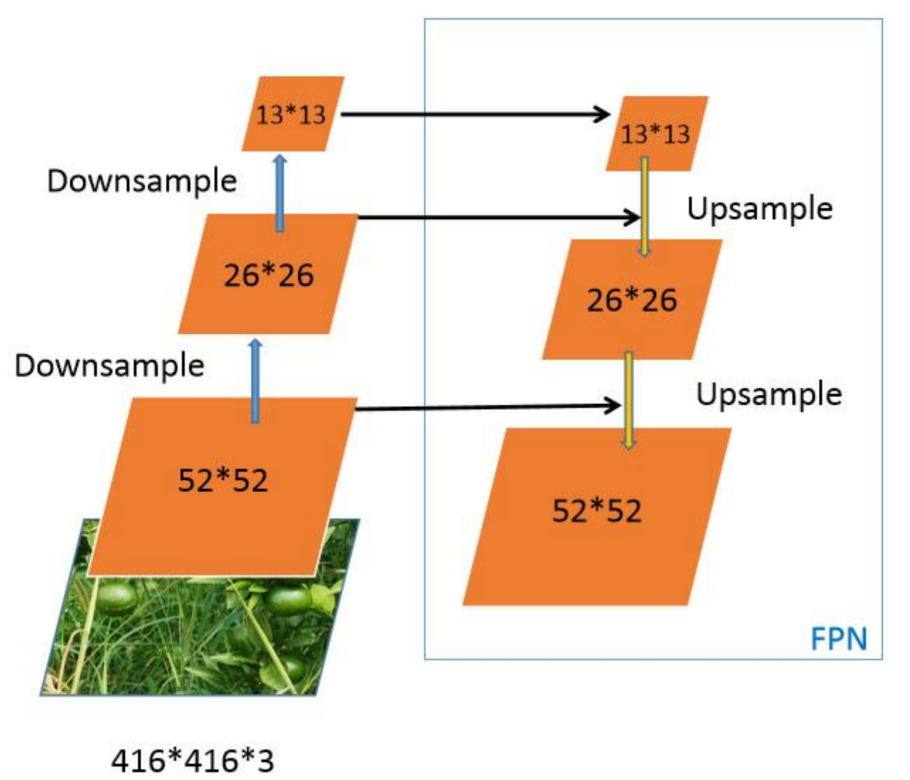

Figure 4. Neck module.

\subsubsection{Output}

Yolov5 used GIoU as the loss function of the bounding box. In the post-processing of target detection, Yolov5 used weighted NMS to filter target boxes.

\subsection{Object Tracking and Counting}

DeepSORT had been proven to be one of the fastest and most robust methods for object tracking and counting [32]. It was originally developed by SORT and used object detection for tracking and effectively correlates the object detection of each frame [33]. When the position of an object changed in different frames, DeepSORT used the Hungarian algorithm to associate with the object in the previous frame [34], allowing the Kalman filter to predict the current position using the last location of the object [35]. Due to the similar size and color of citrus individuals, when the prediction box jumps, the ID of the prediction box represents citrus target changes, causing errors in quantitative statistics. Therefore, we introduced "virtual line" and "virtual region" to accurately predict the number of fruits of citrus fruit trees. Figure 5 shows the flowchart of green citrus counting.

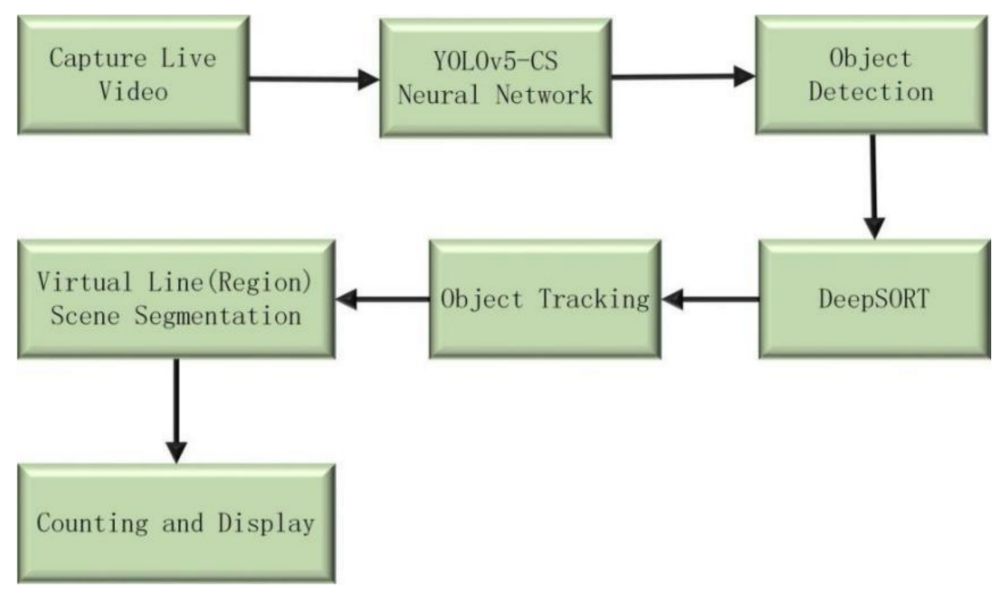

Figure 5. Green citrus counting. 
To count the number of green citrus, we set a virtual line in the video. This divides the scene and detects the center point of the citrus fruit prediction box. If the center point of the citrus prediction box crosses the virtual line, the number is increased by one. Therefore, we only need to walk around the tree so that the number of citrus can be counted. This method provides an efficient and accurate reference for citrus orchard yield estimation. Figure 6 shows the diagram of the virtual line for counting.

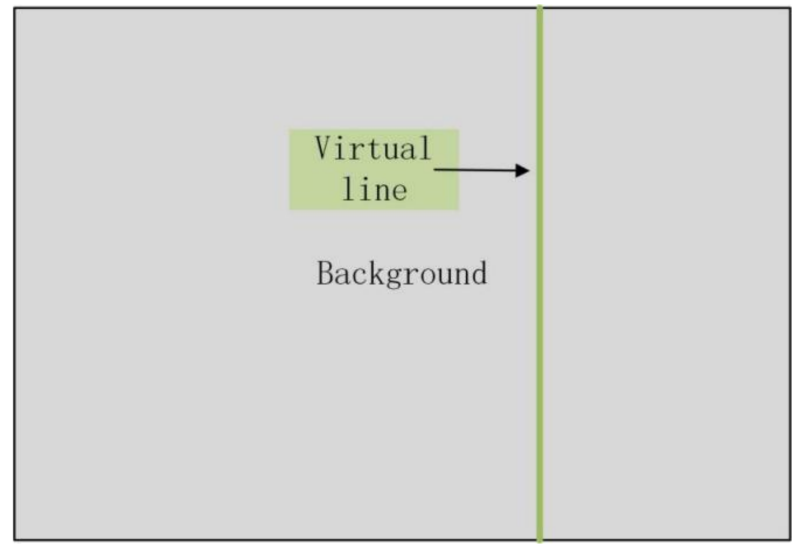

Figure 6. Virtual line for counting.

\section{Materials and Methods}

\subsection{Dataset Preprocessing}

\subsubsection{Data Acquisition}

In this paper, we collected citrus images from citrus orchards in South China Agricultural University, Guangzhou Conghua and Hunan Yizhang, using DJI MAVIC Air2 drones, SLR cameras (Panasonic DMC-G7) and Honor 20 mobile phone for data collection. The shooting time was from 9:00 a.m. to 6:00 p.m. The shooting environment includes natural scenes, such as forward light, backlight, clear and blurred shots under sunny, cloudy, and rainy conditions. The shooting angles are forward, upward, overhead, and multiple angles. We collected more than 3000 original images.

\subsubsection{Data Augmentation and Labeling}

The data were cleaned and filtered from more than 3000 original images and were augmented using data augmentation methods, such as vertical and horizontal mirroring, displacement, blur, rotation $270^{\circ}$, and salt and pepper noise, respectively. Figure 7 provides a view of these data enhancement methods. Finally, the original and augmented images are used as the dataset, containing 2831 images, such that the training and test sets are 2211 and 620, respectively, as shown in Table 1. Figure 8 shows the labeling process of the dataset using labeling software.

\subsection{YOLOv5-CS Model Design and Edge-Computing System Migration \& Deployment \\ 3.2.1. Model Design}

Due to the difficulty of detecting green fruits in the natural environment and the time-consumption of manually taking statistics of citrus yields, this paper proposed a lightweight feasible detection and counting model using the improved YOLOv5-CS model and edge-computing platform. From Figure 9, the model used YOLOv5 as the main model, combined with the improved Conv_CBAM module to replace the original Conv module, which increased the model's attention and feature extraction capabilities for different channels and spaces of the picture without increasing the calculation. Furthermore, it helped to improve the detection accuracy of green citrus. Additionally, Figure 10 shows a small target detection layer. It not only outputs the feature maps of $52^{*} 52,26^{*} 26$, and $13^{*} 13$, but the output with a size of $104^{*} 104$ strengthens the model to recognize small citrus fruits. 


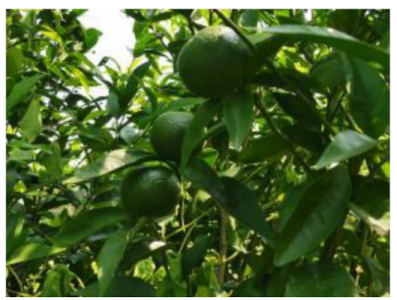

(a)

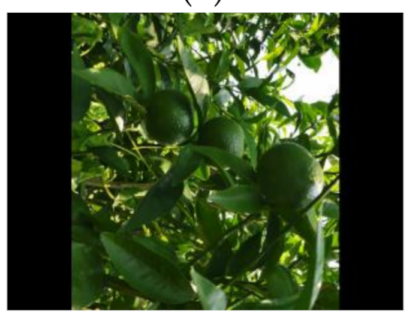

(e)

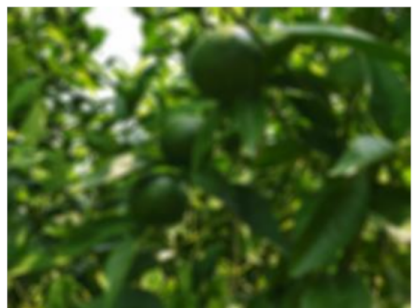

(b)

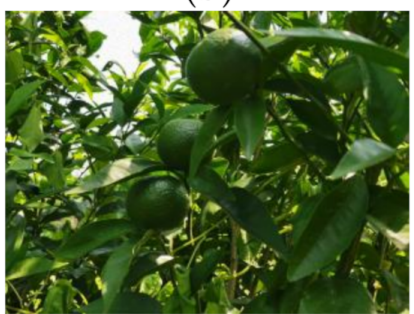

(f)

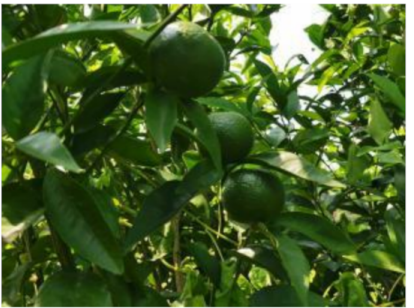

(c)

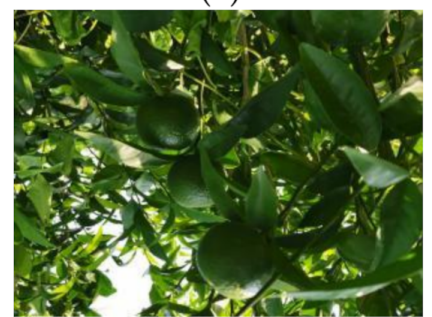

(g)

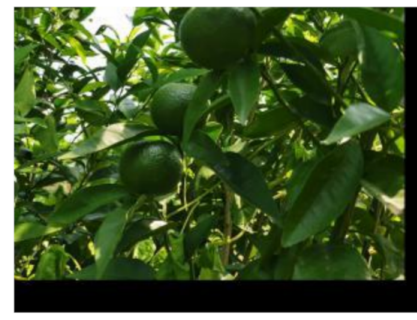

(d)

Figure 7. Data augmentation methods. (a) origin, (b) blur, (c) horizontal mirroring, (d) move, (e) rotate $270^{\circ}$, (f) noise, (g) vertical mirroring.

Table 1. Green citrus dataset.

\begin{tabular}{ccccc}
\hline Dataset & Label & Training Set & Test Set & Total \\
\hline green citrus & unripe citrus & 2211 & 620 & 2831 \\
\hline
\end{tabular}

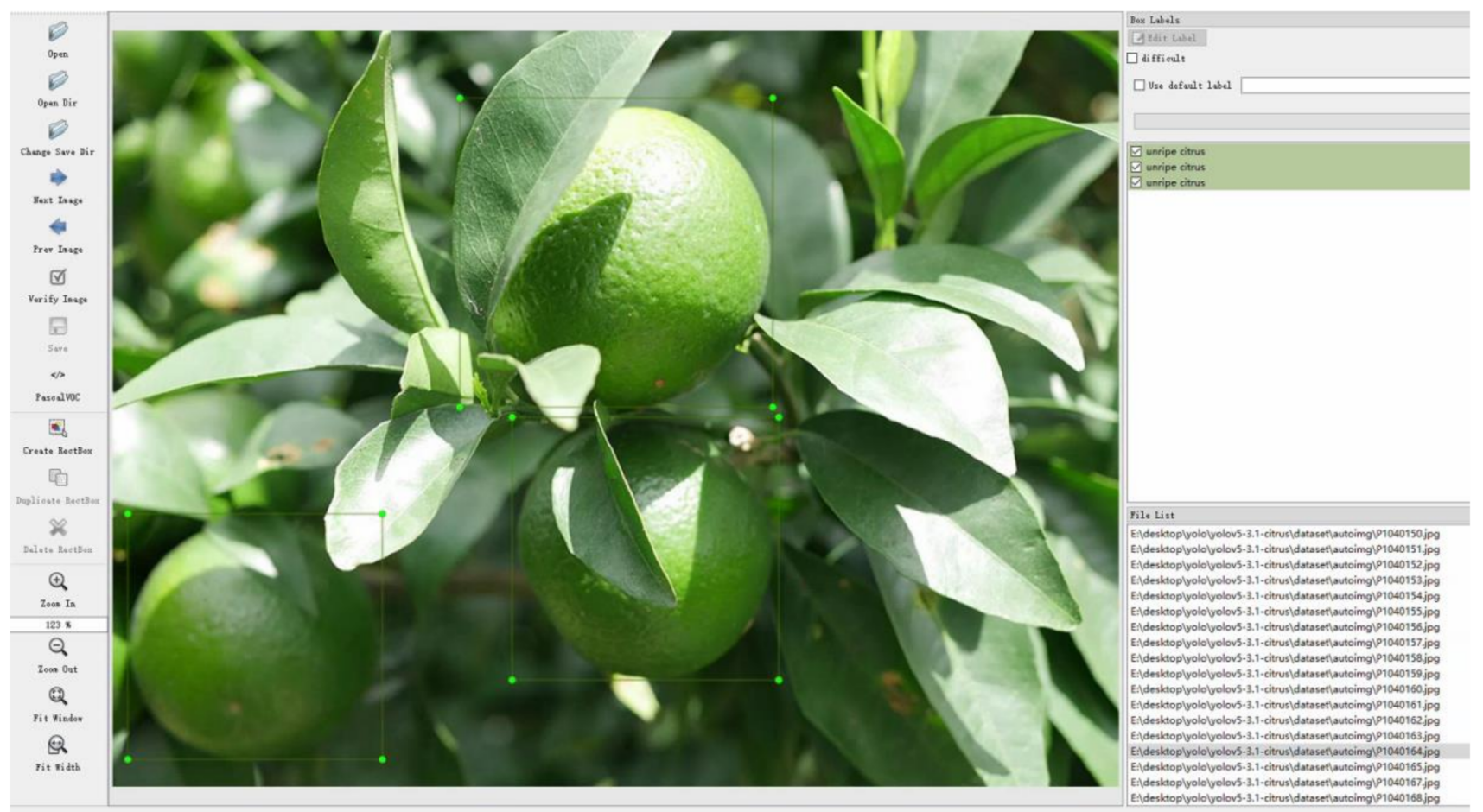

Figure 8. Data labeling. 


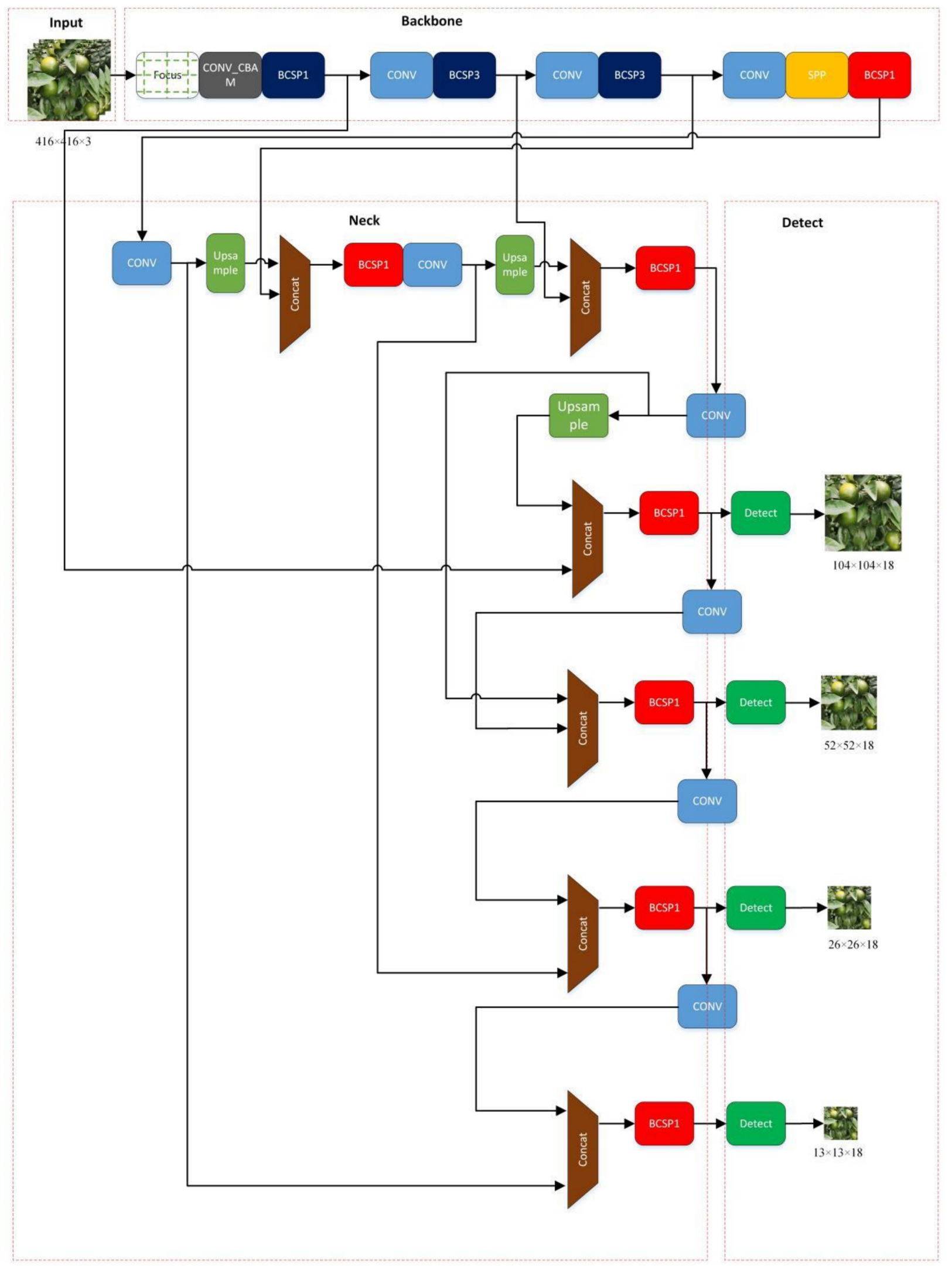

Figure 9. YOLOv5-CS Architecture. 

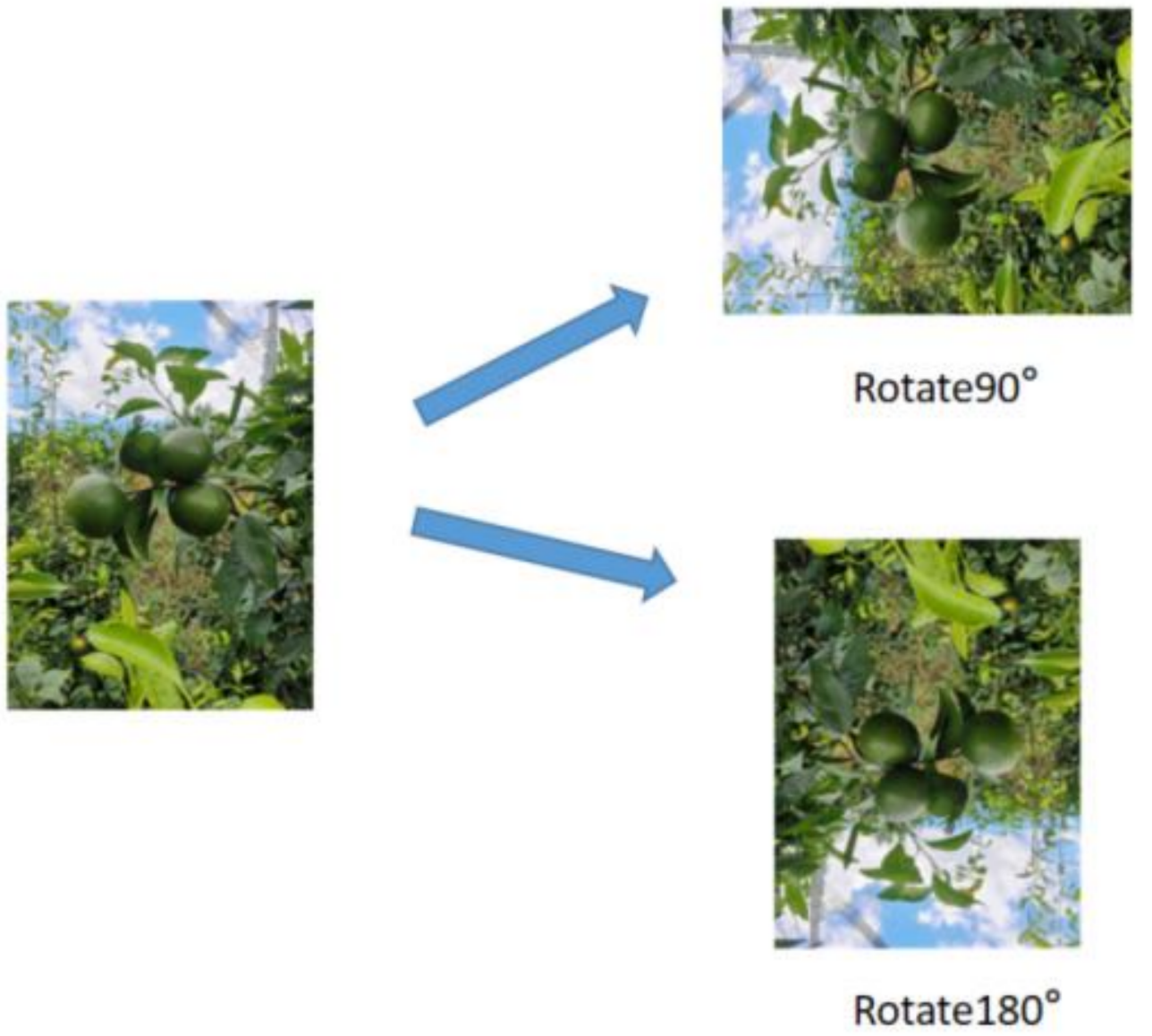

Figure 10. Image rotation.

\subsubsection{Model Optimization}

Because the green citrus is small with a color similar to the leaves, it is difficult for the YOLOv5 model to detect individual small citrus fruits. Thus, we improved the YOLOv5 by adding image rotation codes, a small object detection layer and an attention mechanism to improve the accuracy of the model for green citrus detection.

\section{- Image Rotation}

The YOLOv5 model provided many data augmentation methods. To improve the algorithm's detection accuracy of green citrus from different angles, we added codes for vertical rotation at $90^{\circ}$ and $180^{\circ}$ to the model, effectively enhancing the model's generalization ability. Figure 10 shows the image rotation.

\section{- Small Object Detection Layer}

Due to the size of the green citrus being relatively small, its pixel characteristics are similar to those of leaves under natural light. Therefore, adding modules to improve the detection accuracy and speed of the model is necessary. Thus, we added a small target detection layer and one more up-sampling and down-sampling process to the feature map of the YOLOv5 model. Simultaneously, the acquired feature map is Concat fused with that obtained in the second layer of the backbone network and so the obtained feature map with a larger size is used for small target detection. Table 2 shows the improved YOLOv5-CS network structure, where serial numbers 1 and 17-23 represent the replaced attention mechanism and additional small target detection module, respectively. 
Table 2. YOLOv5-CS network structure.

\begin{tabular}{|c|c|c|c|c|}
\hline Serial Number & From & Params & Module & Arguments \\
\hline 0 & -1 & 3520 & Focus & {$[3,32,3]$} \\
\hline 1 & -1 & 19,170 & Conv_CBAM & {$[32,64,3,2]$} \\
\hline 2 & -1 & 19,904 & BottleneckCSP & {$[64,64,1]$} \\
\hline 3 & -1 & 73,984 & Conv & {$[64,128,3,2]$} \\
\hline 4 & -1 & 161,152 & BottleneckCSP & {$[128,128,3]$} \\
\hline 5 & -1 & 295,424 & Conv & {$[128,256,3,2]$} \\
\hline 6 & -1 & 641,792 & BottleneckCSP & {$[256,256,3]$} \\
\hline 7 & -1 & $1,180,672$ & Conv & {$[256,512,3,2]$} \\
\hline 8 & -1 & 656,896 & SPP & {$[512,512,[5,9,13]]$} \\
\hline 9 & -1 & $1,248,768$ & BottleneckCSP & {$[512,512,1$, False $]$} \\
\hline 10 & -1 & 131,584 & Conv & {$[512,256,1,1]$} \\
\hline 11 & -1 & 0 & Upsample & [None, 2, 'nearest'] \\
\hline 12 & {$[-1,6]$} & 0 & Concat & [1] \\
\hline 13 & -1 & 378,624 & BottleneckCSP & {$[512,256,1$, False $]$} \\
\hline 14 & -1 & 66,048 & Conv & {$[256,128,1,1]$} \\
\hline 15 & -1 & 0 & Upsample & [None, 2, 'nearest'] \\
\hline 16 & {$[-1,4]$} & 0 & Concat & [1] \\
\hline 17 & -1 & 345,856 & BottleneckCSP & {$[384,256,1$, False $]$} \\
\hline 18 & -1 & 33,024 & Conv & {$[256,128,1,1]$} \\
\hline 19 & -1 & 0 & Upsample & [None, 2, 'nearest'] \\
\hline 20 & -1 & 0 & Concat & [1] \\
\hline 21 & -1 & 86,912 & BottleneckCSP & {$[192,128,1$, False $]$} \\
\hline 22 & -1 & 147,712 & Conv & {$[128,128,3,2]$} \\
\hline 23 & -1 & 0 & Concat & [1] \\
\hline 24 & -1 & 95,104 & BottleneckCSP & {$[256,128,1$, False $]$} \\
\hline 25 & -1 & 147,712 & Conv & {$[128,128,3,2]$} \\
\hline 26 & {$[-1,14]$} & 0 & Concat & [1] \\
\hline 27 & -1 & 313,088 & BottleneckCSP & {$[256,256,1$, False $]$} \\
\hline 28 & -1 & 590,336 & Conv & {$[256,256,3,2]$} \\
\hline 29 & {$[-1,10]$} & 0 & Concat & [1] \\
\hline 30 & -1 & $1,248,768$ & BottleneckCSP & {$[512,512,1$, False $]$} \\
\hline
\end{tabular}

\section{- $\quad$ CBAM}

To detect the green citrus from the green leaves in Table 2, after the backbone network focus module, the attention mechanism CONV_CBAM module replaces the original CONV module to obtain more detailed information about the citrus and reduce interference from leaves and complex backgrounds. There are two attention mechanisms: squeeze-andexcitation (SE) and convolutional block attention module (CBAM). The SE module pays attention to the channel information, which mainly solves the loss problem caused by the different weights of different channels in the feature graph. The CBAM module includes both channel and spatial attention modules. The module takes the output of the channel attention module as input for the spatial attention module. After two pooling operations and a convolution operation with a convolution kernel of $7^{*} 7$, the feature graph with the size of $\mathrm{H}^{*} \mathrm{~W}^{*} 2$ is obtained. Spatial attention features are also outputted through the Sigmoid function. The main innovation of the network is that the model can learn the spatial attention features of the output through the relationship between the channel and the space.

\section{- Loss function}

The loss function IoU is a commonly used evaluation indicator in target detection. It evaluates the distance between the predicted box $\mathrm{B}$ of the model and the ground truth of the model. The characteristic of IoU is that it is insensitive to scale. However, when both 
boxes $\mathrm{B}$ and $\mathrm{B}^{\mathrm{gt}}$ do not overlap, the IoU value is zero. Here, there is no gradient return, and no learning or training can be performed.

$$
\begin{gathered}
\mathrm{IoU}=\frac{\left|\mathrm{B} \cap \mathrm{B}^{\mathrm{gt}}\right|}{\left|\mathrm{B} \cup \mathrm{B}^{\mathrm{gt}}\right|}, \\
\mathcal{L}_{\mathrm{IoU}}=1-\frac{\left|\mathrm{B} \cap \mathrm{B}^{\mathrm{gt}}\right|}{\left|\mathrm{B} \cup \mathrm{B}^{\mathrm{gt}}\right|},
\end{gathered}
$$

where $\mathrm{B}$ denotes the predicted box and $\mathrm{B}^{\mathrm{gt}}$ represents ground truth.

To solve this problem, Rezatofighi et al. proposed GloU (generalized IoU) in 2019 [36]. GIoU focuses on areas where the predicted box overlaps the real box and other nonoverlapping areas. When the prediction and real box do not overlap, the prediction box can be prompted to move toward the real box.

$$
\begin{gathered}
\text { GIoU }=\operatorname{IoU}-\frac{\left|\frac{\mathrm{C}}{\mathrm{B} \cup \mathrm{B}^{\mathrm{gt}}}\right|}{|\mathrm{C}|}, \\
\mathcal{L}_{\mathrm{GIOU}}=1-\operatorname{IoU}+\frac{\left|\mathrm{C}-\mathrm{B} \cup \mathrm{B}^{\mathrm{gt}}\right|}{|\mathrm{C}|},
\end{gathered}
$$

where $C$ represents the smallest rectangular box in which $B$ and $B^{\text {gt }}$ can be contained. However, the method still has problems, such as an unstable prediction box and divergence of the training process. To directly minimize the distance between the two boxes for a better convergence, Zheng et al. proposed DIoU (Distance-IoU) and CIoU (Complete IoU) [37]. In contrast, DIoU introduces the distancing mechanism between the center points of the real and predicted boxes. For the horizontal and vertical directions, the DIoU loss converges quickly, and the GIoU loss remains almost the same as the IoU loss. However, the DIoU calculation does not consider the loss of width and height but only finds the overlapping area of the two boxes and the distance between both center points of the predicted and real boxes. However, if the center points of both boxes overlap but the width and height are different, the loss value is unchanged.

$$
\begin{gathered}
\text { DIoU }=\text { IoU }-\frac{\rho^{2}\left(\mathrm{~B}, \mathrm{~B}^{\mathrm{gt}}\right)}{\mathrm{c}^{2}}, \\
\mathcal{L}_{\mathrm{DIoU}}=1-\mathrm{IoU}+\frac{\rho^{2}\left(\mathrm{~B}, \mathrm{~B}^{\mathrm{gt}}\right)}{\mathrm{c}^{2}},
\end{gathered}
$$

where represents the Euclidean distance between $B$ and $B^{g t}$, and $c$ represents the length of the diagonal line containing the smallest box. On this basis, they proposed CloU. CIoU adds an impact factor, which considers the aspect ratio of the predicted box to fit the real box.

$$
\begin{gathered}
\mathcal{L}_{\mathrm{CIOU}}=1-\operatorname{IoU}+\frac{\rho^{2}\left(\mathrm{~B}, \mathrm{~B}^{\mathrm{gt}}\right)}{\mathrm{c}^{2}}+\alpha v, \\
v=\frac{4}{\pi^{2}}\left(\tan ^{-1} \frac{\mathrm{w}^{\mathrm{gt}}}{\mathrm{h}^{\mathrm{gt}}}-\tan ^{-1} \frac{\mathrm{w}}{\mathrm{h}}\right)^{2}, \\
\alpha=\frac{v}{(1-\mathrm{IoU})+v^{\prime}},
\end{gathered}
$$

where $\alpha$ is a positive tradeoff parameter, and $v$ measures the consistency of the aspect ratio.

\subsubsection{Edge-Computing System Migration \& Deployment}

The Jetson Xavier NX edge-computing platform based on ARM architecture and produced by NVIDIA was applied in this paper. The platform is small, approximately 70 
$\times 45 \mathrm{~mm}$ in size, and brings the performance of supercomputers to the edge through the system of modules. The platform includes a 6-core Carmel ARM CPU, 384 NVIDIA CUDA ${ }^{\circledR}$ Cores, 48 Tensor Cores, and two NVIDIA deep learning accelerator (NVDLA) engines, which can provide up to 21 TOPS of computing power. Camera, displayer, and portable power devices were added to form an edge-computing system platform based on the edge-computing platform. Figure 11 shows the edge-computing system platform diagram.

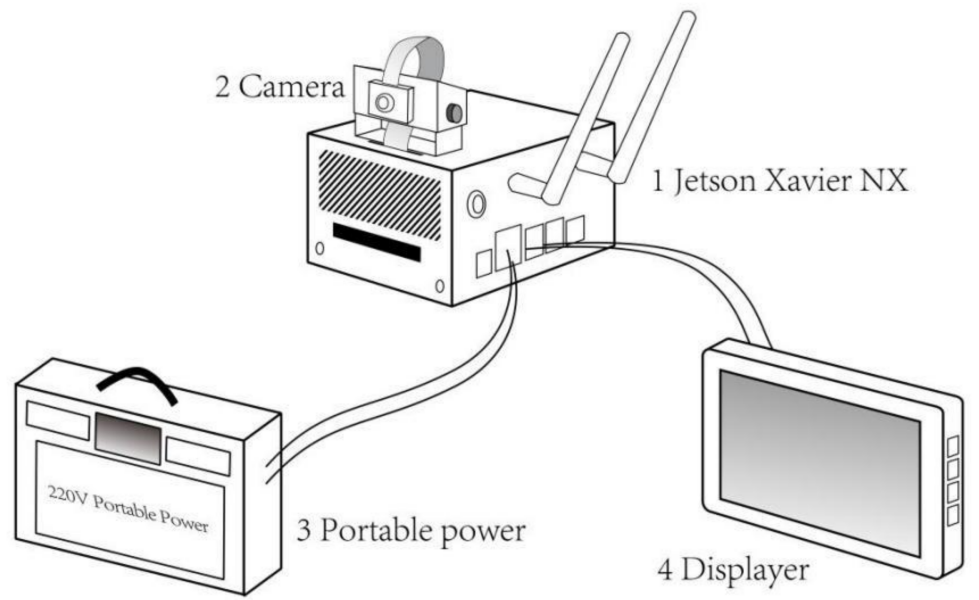

Figure 11. Edge-computing system platform.

To facilitate the deployment of the YOLOv5-CS model, it is necessary to deploy the operating environment on Jetson Xavier NX and call the hardware performance of the platform, especially the deep learning accelerator engine. To achieve production statistics, USB and HDMI interfaces and expansion pins of the platform were used. The flowchart of counting the number of green citrus is shown in Figure 12. The edge-computing platform obtained the video stream in a short time by calling the high-definition camera and transmitting the video stream information into the RAM. The CPU control modules, such as CUDA cores, Tensor cores, and NVDLA, use heterogeneous parallel computing to accelerate the model by hardware. The detection result, as well as the number of citrus, were displayed using the visualization module.

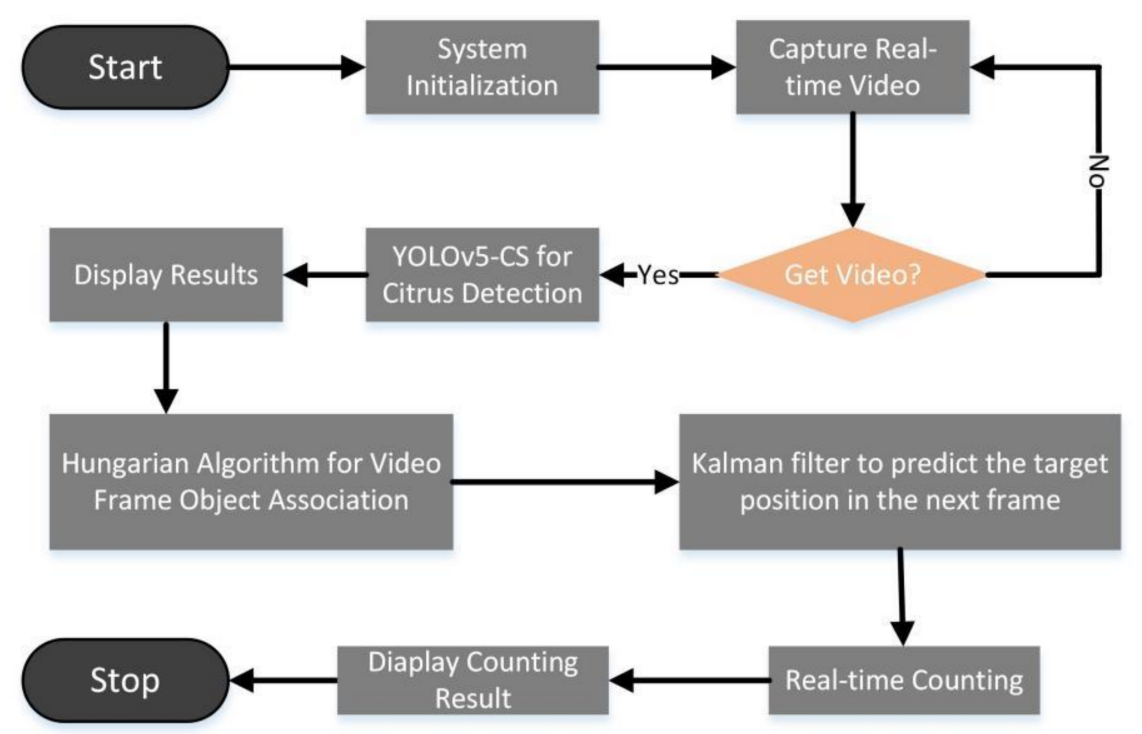

Figure 12. Green citrus counting. 


\subsection{Learning Rate}

The learning rate affects the convergence speed of the YOLOv5-CS model. Here we used the cosine annealing algorithm to dynamically change the learning rate. In the warmup stage, one-dimensional linear interpolation was used to update the learning rate of each iteration, after which the cosine annealing algorithm was used to update the learning rate. The cosine annealing mechanism uses a cosine function to reduce the learning rate. This descent mode is matched with the learning rate. Increasing the learning rate can effectively prevent the model from falling into the local minimum and then train in the direction of the global minimum.

$$
\eta_{\mathrm{t}}=\eta_{\text {min }}^{\mathrm{i}}+\frac{1}{2}\left(\eta_{\text {max }}^{\mathrm{i}}-\eta_{\text {min }}^{\mathrm{i}}\right)\left(1+\cos \left(\frac{\mathrm{T}_{\text {cur }}}{\mathrm{T}_{\mathrm{i}}} \pi\right)\right),
$$

where $\eta_{\min }^{\mathrm{i}}$ and $\eta_{\max }^{\mathrm{i}}$, respectively, represent the minimum and maximum of the learning rate, and define the learning rate range. $\mathrm{T}_{\text {cur }}$ denotes the number of epochs executed since the last training restart, although it is updated after each batch is run. When an epoch has not been executed, the value of $T_{\text {cur }}$ can be a decimal. $T_{i}$ denotes the total number of epochs in the $\mathrm{i}$-th training session.

\subsection{Experiment Platform}

Server-side: Windows 10, Core i9-11900@5.20 GHz CPU, 32 GB RAM, NVIDIA GeForce RTX 3080(10 GB) GPU. AI edge system side: 6-core NVIDIA Carmel ARM ${ }^{\circledR}$ v8.2 64-bit CPU. The model framework is Pytorch, with related software CUDA 11.1, cudnn 11.1, and Python 3.8.10. The original YOLOv5 model and the improved YOLOv5-CS model use YOLOv5s.pt for pretraining and retraining based on the pretraining results. The parameters are set as follows: image input size: $416^{*} 416$, epoch: 50 , initial learning rate: 0.01 , bbox_iou: CIoU.

\subsection{Evaluation Index}

Since the background environment can be identified as citrus or missed during the detection, the accuracy and recall ratios are used to describe the citrus detection. The accuracy and recall rates are given as follows:

$$
\begin{aligned}
& \text { Precision }=\frac{\mathrm{TP}}{\mathrm{TP}+\mathrm{FP}^{\prime}} \\
& \text { Recall }=\frac{\mathrm{TP}}{\mathrm{TP}+\mathrm{FN}^{\prime}}
\end{aligned}
$$

where TP and FP denote positive and negative samples, respectively, predicted to be true, and FN represents a positive sample predicted to be false. By setting the accuracy and recall rate to the vertical and horizontal axes, respectively, gives the accuracy-recall rate curve, referred to as the P-R curve.

To better evaluate the effect of the model, the AP (average precision) of a single category was proposed as the sum of the AP values of each category, which is used to obtain $\mathrm{mAP}$ (mean average precision). The definitions of $\mathrm{AP}$ and $\mathrm{mAP}$ are as follows:

$$
\begin{aligned}
\mathrm{AP} & =\int_{0}^{1} \mathrm{P}(\mathrm{R}) \mathrm{dR}, \\
\mathrm{mAP} & =\frac{\sum_{\mathrm{q}=1}^{\mathrm{Q}} \mathrm{AP}(\mathrm{q})}{\mathrm{Q}},
\end{aligned}
$$

where $Q$ is the number of categories. 


\section{Experiment and Results}

\subsection{Ablation Experiment}

To prove the effectiveness of our model in this paper, we conducted the ablation experiment on both data augmentation processing methods and improved architecture, as shown in Table 3. $\sqrt{ }$ represents using the methods or module.

Table 3. Model ablation experiment.

\begin{tabular}{|c|c|c|c|c|c|}
\hline $\begin{array}{c}\text { Data } \\
\text { Augmentation }\end{array}$ & $\begin{array}{c}\text { Small Object } \\
\text { Detection Layer }\end{array}$ & CBAM & mAP@.5 & Recall & Epochs \\
\hline & & & $96.66 \%$ & $92.74 \%$ & \multirow{4}{*}{100} \\
\hline$\sqrt{ }$ & & & $97.51 \%$ & $96.16 \%$ & \\
\hline$\sqrt{ }$ & $\sqrt{ }$ & & $97.59 \%$ & $96.09 \%$ & \\
\hline$\sqrt{ }$ & $\sqrt{ }$ & $\sqrt{ }$ & $98.05 \%$ & $97.38 \%$ & \\
\hline
\end{tabular}

From the table above, it can be seen that the detection accuracy was improved from $96.66 \%$ to $97.51 \%$ by using data augmentation methods, and the recall was improved by $3.42 \%$. After adding the small object detection layer, the detection accuracy reached $97.59 \%$. The detection accuracy of the model finally achieved $98.05 \%$ after adding CBAM.

\subsection{Training Result}

The performance of the detection model is evaluated using the loss function curve and the mAP@.5. The loss function curve shows the network model's convergence speed and degree of convergence during the training process. Figure 13 shows the loss function curve of the YOLOv5 and improved YOLOv5-CS models.

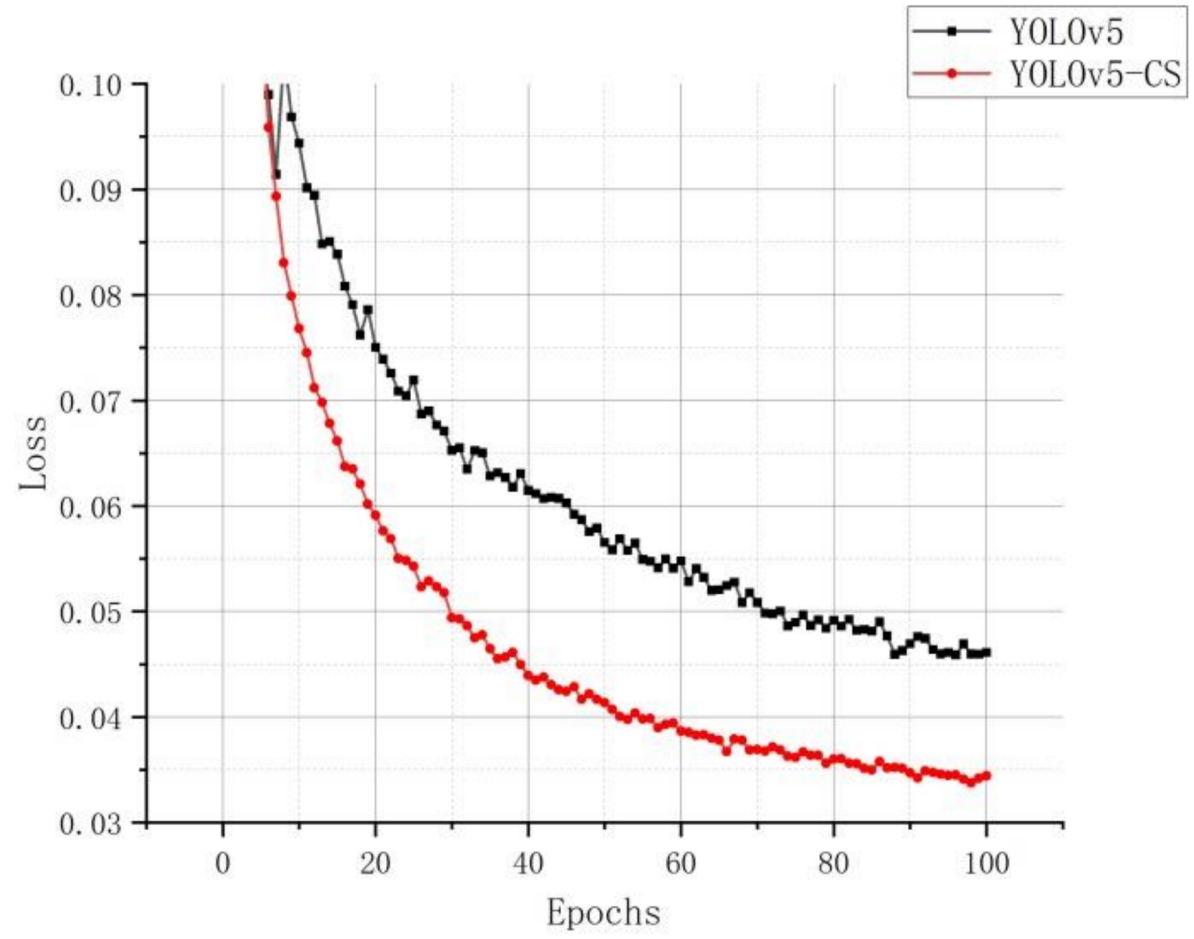

Figure 13. Loss curve.

From the figure above, the improved YOLOv5-CS model curve converged with a faster degree of convergence and a smaller loss value in the training process of 100 epochs. When it finished, the loss value of the improved YOLOv5-CS model was 21.74\% lower than that of the YOLOv5 model, which proves that the former has better convergence. Although the 
loss value has achieved good results, there are still some pictures in the test set that are missed or encountered errors due to excessive occlusion and light, as shown in Figure 14.

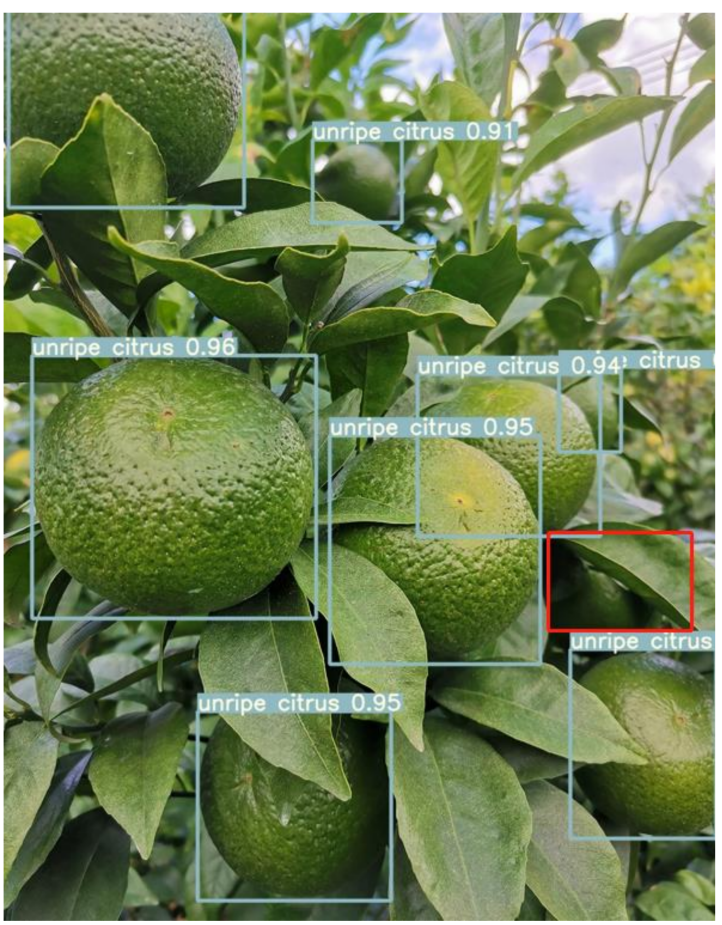

Figure 14. Leakage detection of citrus.

mAP@.5 was used to measure the detection effect of the model. The higher its value, the better the detection effect of the model. Figure 15 shows that, after 100 epochs, the mAP@.5 value of the improved YOLOv5-CS model gradually stabilized, reaching 98.05\%, and the highest during the period reached $98.5 \%$. This shows that the improved YOLOv5CS model has a better result for detecting green citrus and has reached the expectation of accurate recognition.

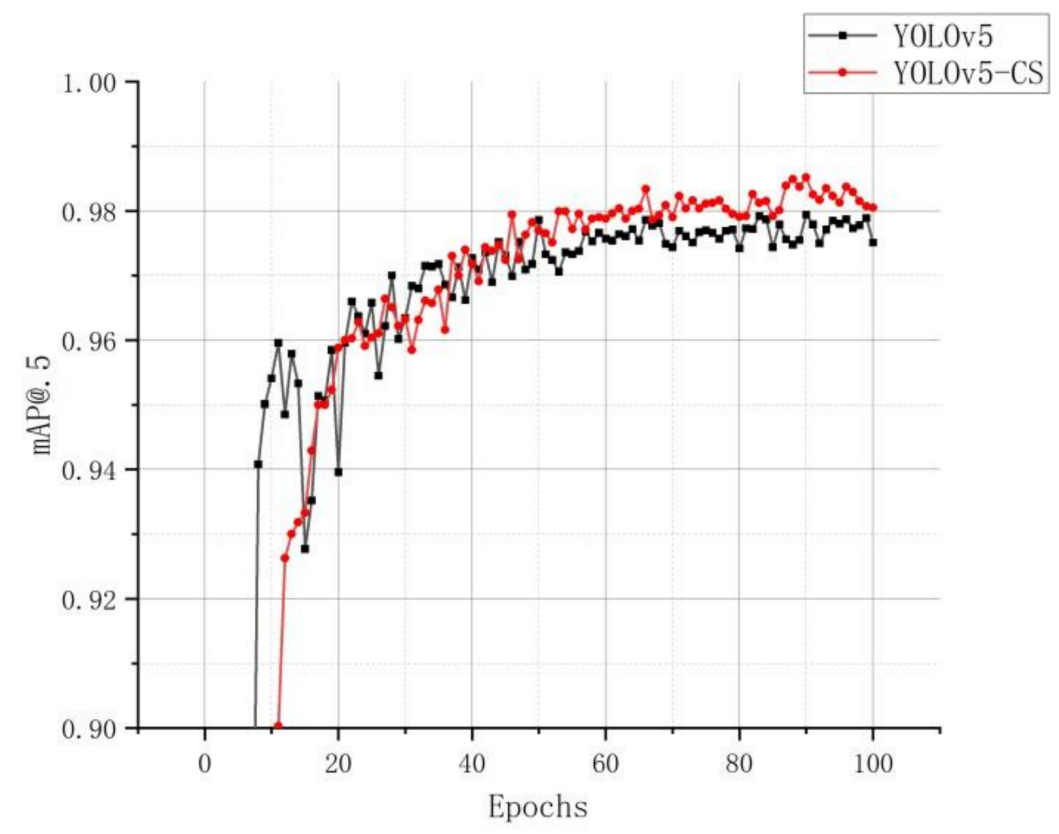

Figure 15. mAP@.5 curve. 
The improved YOLOv5-CS model and the YOLOv5 model were trained for the first time with 100 epochs, respectively, and the improved YOLOv5-CS model was then retraining with 50 epochs; the parameters obtained are shown in Table 4 . Although the precision value of the improved YOLOv5-CS model decreased, the final values of mAP@.5 and recall were $98.23 \%$ and $97.66 \%$, respectively, and the detection time for 620 images in the validation set was $1.61 \mathrm{~s}$ less than that of the YOLOv5 model.

Table 4. Training result.

\begin{tabular}{cccccc}
\hline Neural Network & Epochs & mAP@.5 & Image-Size & Precision & Recall \\
\hline & \multicolumn{5}{c}{ First training } \\
YOLOv5 & 100 & $97.51 \%$ & 416 & $89.81 \%$ & $96.16 \%$ \\
YOLOv5-CS & 100 & $98.05 \%$ & 416 & $84.49 \%$ & $97.38 \%$ \\
& & Retraining & & \\
YOLOv5 & 50 & $97.79 \%$ & 416 & $93.03 \%$ & $95.27 \%$ \\
YOLOv5-CS & 50 & $98.23 \%$ & 416 & $86.97 \%$ & $97.66 \%$ \\
\hline
\end{tabular}

\subsection{Counting Result}

By setting a virtual line on the image screen, the citrus tracked and identified will be detected and counted when passing the line. However, the citrus prediction box may flicker when passing through the line; therefore, to accurately count the number of citrus, we expand the virtual line into a virtual region to ensure citrus detection through this area and improve the accuracy of citrus yield statistics. The diagram of the virtual region in the orchard environment is shown in Figure 16.

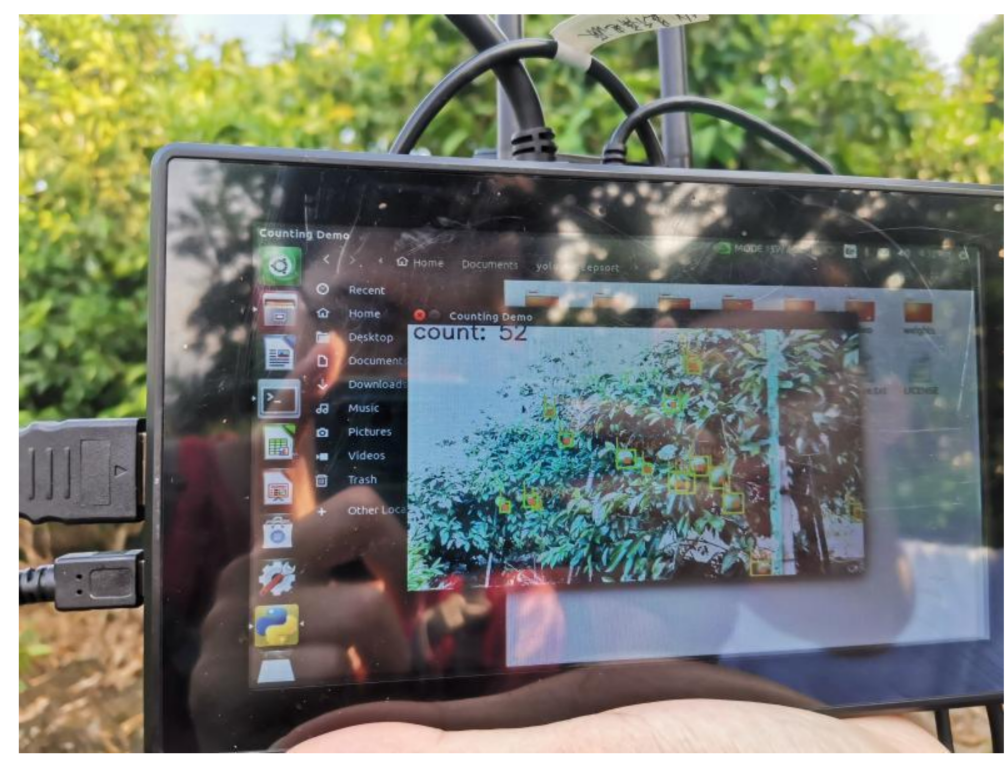

Figure 16. Counting the number of citrus in the orchard.

We conducted experiments on the simulated citrus trees and the real citrus trees. Standing at a distance of $1.5 \mathrm{~m}$ from the citrus trees, the camera was located at half the height of each citrus tree. The counting results of citrus are shown in Tables 5 and 6 . The average relative error of the count of the simulated citrus tree was $4.25 \%$, and the average relative error of the count of the real citrus tree was $8.75 \%$. The main reason of the different errors mainly came from the complexity of the orchard environment, which caused false detection. 
Table 5. Counting results of simulated citrus tree.

\begin{tabular}{cccccc}
\hline $\begin{array}{c}\text { Number of } \\
\text { Experiments }\end{array}$ & $\begin{array}{c}\text { Actual } \\
\text { Number }\end{array}$ & $\begin{array}{c}\text { Number of } \\
\text { Tests }\end{array}$ & $\begin{array}{c}\text { Relative } \\
\text { Error }\end{array}$ & $\begin{array}{c}\text { Average Relative } \\
\text { Error of Ten Times }\end{array}$ & FPS \\
\hline 1 & & 40 & $0 \%$ & \\
2 & 37 & $7.5 \%$ & \\
3 & 36 & $10 \%$ & \\
4 & & 38 & $5 \%$ & \\
5 & 40 & 38 & $5 \%$ & \\
6 & & 39 & $2.5 \%$ & \\
7 & 37 & $7.5 \%$ & \\
8 & 39 & $2.5 \%$ & \\
9 & 40 & $0 \%$ & \\
10 & 39 & $2.5 \%$ & \\
\hline
\end{tabular}

Table 6. Counting results of real citrus tree.

\begin{tabular}{cccccc}
\hline $\begin{array}{c}\text { Number of } \\
\text { Experiments }\end{array}$ & $\begin{array}{c}\text { Actual } \\
\text { Number }\end{array}$ & $\begin{array}{c}\text { Number of } \\
\text { Tests }\end{array}$ & $\begin{array}{c}\text { Relative } \\
\text { Error }\end{array}$ & $\begin{array}{c}\text { Average Relative } \\
\text { Error of Ten Times }\end{array}$ & FPS \\
\hline 1 & & 22 & $8.33 \%$ & & \\
2 & 21 & $12.5 \%$ & \\
3 & 23 & $4.17 \%$ & \\
4 & & 25 & $4.17 \%$ & & \\
5 & 24 & 21 & $12.5 \%$ & \\
6 & & 20 & $16.67 \%$ & \\
7 & 26 & $8.33 \%$ & \\
8 & 22 & $8.33 \%$ & \\
9 & & $4.17 \%$ & & \\
10 & & $8.33 \%$ & & \\
\hline
\end{tabular}

\section{Discussion}

In this paper, we proposed the YOLOv5-CS model with improved robustness and stability in a complex natural orchard environment using the data augmentation method. Additionally, we applied the CBAM module to make the model focus on important feature information and ignore unimportant information. Furthermore, a detection layer was embedded to improve the model's detection accuracy for little citruses. Additionally, using the CIoU as the loss function, it achieved the fastest convergence speed and best convergence effect function. The cosine annealing algorithm was applied to change the learning rate when the model fell into the local optimal solution, thereby training it towards the global optimal solution. Furthermore, the retraining method was used to improve the mAP@.5 and recall values of the model, thereby improving the convergence speed of the model. These improvement methods produced excellent detection results. The inference speeds of YOLOv5s and YOLOv5-CS detecting a picture on the server were $0.018 \mathrm{~s}$ and $0.017 \mathrm{~s}$ respectively, and the inference speeds of those on the Nvidia Jetson Xavier NX were $0.04 \mathrm{~s}$ and $0.037 \mathrm{~s}$ respectively. Next, we used DeepSORT for citrus object tracking and counting. This involved tracking the location of citrus targets by combining the Kalman filtering with the Hungarian algorithm for frame-by-frame analysis of the video. Additionally, we introduced "virtual line" and "virtual region" to improve the accuracy of the citrus target count of the model and avoid duplicate counts. We also migrated the model to the Jetson Xavier NX edge-computing platform to help farmers count the number of green citrus. High-definition cameras were used to capture videos and showed the counting result on a visualization module. Finally, we realized the feasible detection of green citrus and counting on the mobile platform.

If the green citrus detection and counting model could apply to the entire orchard, the rich dataset will help identify citrus at different growth stages and collect several fruits, which can construct various fruits detection and counting systems for detecting and 
counting different fruits simultaneously. Hence, our system will have more functional applications in the intelligent orchard.

\section{Conclusions and Future Works}

Recently, there has been rapid development in object detection and multi-object tracking with great interest in their applications in agriculture. Briefly, the contributions of this paper are as follows. Here, an improved YOLOv5-CS model was proposed as an extension of the YOLOv5 model. Our model adopted object detection and multi-object tracking technology, to achieve feasible detection and counting of green citrus in orchards. Codes for image rotation were added to data augmentation. The CBAM module and the small-object detection layer were embedded in the backbone network. The CIoU loss function and cosine annealing algorithm were introduced in the training. We migrated the improved YOLOv5-CS model to the edge-computing platform to help farmers use mobile devices to detect and count green citrus, which is of great reference significance for citrus yield estimation. Until now, this is the first time in the computer field that the object detection and counting of citrus were applied to the edge-computing platform and the "virtual region" was introduced to realize the counting of green citrus in the orchard. Compared with the original YOLOv5 model, the mAP@.5 and recall values of the improved model improved by $0.72 \%$ and $1.50 \%$, respectively. The inference speeds of YOLOv5s and YOLOv5-CS detecting a picture on the server were $0.018 \mathrm{~s}$ and $0.017 \mathrm{~s}$ respectively, and the inference speeds of those on Nvidia Jetson Xavier NX were $0.04 \mathrm{~s}$ and $0.037 \mathrm{~s}$ respectively. The detection and counting frame rate of the video on the edge-computing platform was 28 FPS. It also had strong robustness in the complex orchard environment, providing farmers with portable and intelligent citrus counting equipment, thus reducing planting costs caused by manual counting. In the future, we will continue to improve the prediction accuracy and speed, especially that of citrus at different growth stages. Nowadays, yield estimation of fruits and automated picking have become important development directions for orchard management and production. We will also study the deployment of citrus counting equipment on drones, study autonomous navigation and synchronized positioning using SLAM and visual navigation technologies, and contribute to the development of intelligent orchards.

Author Contributions: Conceptualization, S.L. (Shilei Lyu) and Z.L.; methodology, S.L. (Shilei Lyu) and R.L.; software, Y.Z.; validation, S.L. (Shilei Lyu), R.L. and Y.Z.; formal analysis, R.L.; data curation, S.L. (Shilei Lyu) and R.L.; writing-original draft preparation, S.L. (Shilei Lyu) and R.L.; writing-review and editing, Z.L.; visualization, R.F. and S.L. (Siying Liu); supervision, Z.L.; funding acquisition, Z.L. All authors have read and agreed to the published version of the manuscript.

Funding: This research was funded by Guangdong Laboratory for Lingnan Modern Agriculture Project (NT2021009); Guangdong Natural Science Foundation (2021A1515010923); National Natural Science Foundation of China (31971797 and 61601189); Special projects for key fields of colleges and universities in Guangdong Province (2020ZDZX3061); China Agriculture Research System of MOF and MARA (CARS-26); Subtopics of National Key R\&D Program Projects (2020YFD1000107).

Institutional Review Board Statement: Not applicable.

Informed Consent Statement: Not applicable.

Data Availability Statement: Not applicable.

Acknowledgments: The authors would like to thank anonymous reviewers for their criticism and suggestions. We would also like to thank Difang Deng for research data support.

Conflicts of Interest: The authors declare no conflict of interest.

\section{References}

1. Koirala, A.; Walsh, K.B.; Wang, Z.; McCarthy, C. Deep learning-Method overview and review of use for fruit detection and yield estimation. Comput. Electron. Agric. 2019, 162, 219-234. [CrossRef]

2. Yi, S.; Li, J.J.; Zhang, P.; Wang, D.D. Detecting and counting of spring-see citrus using YOLOv4 network model and recursive fusion of features. Trans. Chin. Soc. Agric. Eng. 2020, 29, 15-20. 
3. Wu, X.; Qi, Z.Y.; Wang, L.J.; Yang, J.J.; Xia, X. Apple Detection Method Based on Light-YOLOv3 Convolutional Neural Net-work. Chin. Soc. Agric. Mach. 2020, 51, 17-25.

4. Chen, W.K.; Lu, S.L.; Liu, B.H.; Li, G.; Liu, X.Y.; Chen, M. Real-time Citrus Recognition under Orchard Environment by Im-proved YOLOv4. J. Guangxi Nor. Univ. (Nat. Sci. Ed.) 2021, 39, 134-146.

5. $\quad$ Li, Z.J.; Yang, S.H.; Shi, D.S.; Liu, X.X.; Zheng, Y.J. Yield Estimation Method of Apple Tree Based on Improved Lightweight YOLOv5. Smart Agric. 2021, 3, 100-114.

6. Li, X.; Qin, Y.; Wang, F.; Guo, F.; Yeow, J.T.W. Pitaya detection in orchards using the MobileNet-YOLO model. In Proceedings of the 2020 39th Chinese Control Conference (CCC), Shenyang, China, 27-29 July 2020; IEEE: Shenyang, China, 2020.

7. Bi, S.; Gao, F.; Chen, J.W.; Zhang, L. Detection Method of Citrus Based on Deep Convolution Neural Network. Trans. Chin. Soc. Agric. Mach. 2019, 50, 181-186.

8. Xiong, J.T.; Zheng, Z.H.; Liang, J.E.; Liu, B.L.; Sun, B.X. Citrus Detection Method in Night Environment Based on Improved YOLO v3 Network. Chin. Soc. Agric. Mach. 2020, 51, 199-206.

9. Li, S.J.; Hu, D.Y.; Gao, S.M.; Lin, J.H.; An, X.S.; Zhu, M. Real-time Classification and Detection of Citrus Based on Improved Single Short Multibox Detecter. Trans. Chin. Soc. Agric. Eng. 2019, 35, 307-313.

10. Lyu, S.L.; Lu, S.H.; Li, Z.; Hong, T.S.; Xue, Y.J.; Wu, B.L. Orange Recognition Method Using Improved YOLOv3-LITE Light-weight Neural Network. Trans. Chin. Soc. Agric. Eng. 2019, 35, 205-214.

11. Zheng, Z.; Xiong, J.; Lin, H.; Han, Y.; Sun, B.; Xie, Z.; Yang, Z.; Wang, C. A Method of Green Citrus Detection in Natural Environments Using a Deep Convolutional Neural Network. Front. Plant Sci. 2021, 12, 1861-1874. [CrossRef]

12. Kuznetsova, A.; Maleva, T.; Soloviev, V. Detecting Apples in Orchards Using YOLOv3 and YOLOv5 in General and CloseUp Images. In International Symposium on Neural Networks; Springer: Cham, Switzerland; Berlin/Heidelberg, Germany, 2020; pp. 233-243.

13. Parico, A.; Ahamed, T. Real Time Pear Fruit Detection and Counting Using YOLOv4 Models and Deep SORT. Sensors 2021, 21, 4803. [CrossRef] [PubMed]

14. Yan, B.; Fan, P.; Lei, X.; Liu, Z.; Yang, F. A Real-Time Apple Targets Detection Method for Picking Robot Based on Improved YOLOv5. Remote Sens. 2021, 13, 1619. [CrossRef]

15. Chen, W.; Lu, S.; Liu, B.; Li, G.; Qian, T. Detecting Citrus in Orchard Environment by Using Improved YOLOv4. Sci. Program. 2020, 2020, 8859237. [CrossRef]

16. Xue, Y.J.; Huang, N.; Tu, S.Q.; Mao, L.; Yang, A.Q.; Zhu, X.M.; Yang, X.F.; Chen, P.F. Immature Mango Detection Based on Improved YOLOv2. Trans. Chin. Soc. Agric. Eng. 2018, 34, 173-179.

17. Shi, R.; Li, T.; Yamaguchi, Y. An attribution-based pruning method for real-time mango detection with YOLO network. Comput. Electron. Agric. 2020, 169, 105214. [CrossRef]

18. $\mathrm{Mu}, \mathrm{Y}$;; Chen, T.-S.; Ninomiya, S.; Guo, W. Intact Detection of Highly Occluded Immature Tomatoes on Plants Using Deep Learning Techniques. Sensors 2020, 20, 2984. [CrossRef] [PubMed]

19. Wang, D.; He, D. Channel pruned YOLO V5s-based deep learning approach for rapid and accurate apple fruitlet detection before fruit thinning. Biosyst. Eng. 2021, 210, 271-281. [CrossRef]

20. Song, S.; Li, Y.; Huang, Q.; Li, G. A New Real-Time Detection and Tracking Method in Videos for Small Target Traffic Signs. Appl. Sci. 2021, 11, 3061. [CrossRef]

21. Yao, J.; Qi, J.; Zhang, J.; Shao, H.; Yang, J.; Li, X. A Real-Time Detection Algorithm for Kiwifruit Defects Based on YOLOv5. Electronics 2021, 10, 1711. [CrossRef]

22. Janowski, A.; Kaźmierczak, R.; Kowalczyk, C.; Szulwic, J. Detecting Apples in the Wild: Potential for Harvest Quantity Es-timation. Sustainability 2021, 13, 8054. [CrossRef]

23. Xu, D.G.; Wang, L.; Li, F. Review of Typical Object Detection Algorithms for Deep Learning. Comput. Eng. Appl. 2021, 57, 10-25.

24. Redmon, J.; Divvala, S.; Girshick, R.; Farhadi, A. You Only Look Once: Unified, Real-Time Object Detection. In Proceedings of the 2016 IEEE Conference on Computer Vision and Pattern Recognition (CVPR), Las Vegas, NV, USA, 27-30 June 2016; IEEE: Piscataway, NJ, USA, 2016; pp. 779-788. [CrossRef]

25. Redmon, J.; Farhadi, A. YOLO9000: Better, Faster, Stronger. In Proceedings of the 30th IEEE Conference on Computer Vision and Pattern Recognition, Honolulu, HI, USA, 21-26 July 2017; IEEE: Piscataway, NJ, USA, 2017; pp. 6517-6525. [CrossRef]

26. Redmon, J.; Farhadi, A. YOLOv3: An Incremental Improvement, Computer Vision and Pattern Recognition (CVPR). arXiv 2018, arXiv:1804.02767.

27. Bochkovskiy, A.; Wang, C.Y.; Liao, H.Y.M. YOLOv4 Optimal Speed and Accuracy of Object Detection. In Proceedings of the Computer Vision and Pattern Recognition. arXiv 2020, arXiv:2004.10934.

28. YOLOv5. Available online: https://github.com/ultralytics/yolov5 (accessed on 10 June 2020).

29. Wang, C.Y.; Liao, H.Y.M.; Wu, Y.H.; Chen, P.Y.; Hsieh, J.W.; Yeh, I.H. CSPNet: A New Backbone that can Enhance Learning Capability of CNN. In Proceedings of the 2020 IEEE/CVF Conference on Computer Vision and Pattern Recognition Workshops (CVPRW), Seattle, WA, USA, 14-19 June 2020; IEEE: Piscataway, NJ, USA, 2020; pp. 1571-1580.

30. Luvizon, D.; Tabia, H.; Picard, D. SSP-Net: Scalable Sequential Pyramid Networks for Real-Time 3D Human Pose Regression. arXiv 2020, arXiv:2009.01998. 
31. Lin, T.; Dollár, P.; Girshick, R.B.; He, K.; Hariharan, B.; Belongie, S. Feature Pyramid Networks for Object Detection. In Proceedings of the IEEE Conference on Computer Vision and Pattern Recognition, Honolulu, HI, USA, 21-26 July 2017; IEEE: Piscataway, NJ, USA, 2017. [CrossRef]

32. Wojke, N.; Bewley, A.; Paulus, D. Simple online and realtime tracking with a deep association metric. In Proceedings of the 2017 IEEE International Conference on Image Processing (ICIP), Beijing, China, 17-20 September 2017; IEEE: Piscataway, NJ, USA, 2017; pp. 3645-3649.

33. Bewley, A.; Ge, Z.; Ott, L.; Ramos, F.; Upcroft, B. Simple Online and Realtime Tracking. In Proceedings of the International Conference on Image Processing (ICIP), Phoenix, AZ, USA, 25-28 September 2016; IEEE: Piscataway, NJ, USA, 2016.

34. Kuhn, H.W. The Hungarian method for the assignment problem. Nav. Res. Logist. (NRL) 2005, 52, 7-21. [CrossRef]

35. Kalman, R.E. A New Approach to Linear Filtering and Prediction Problems. J. Basic Eng. 1960, 82, 35-45. [CrossRef]

36. Rezatofighi, H.; Tsoi, N.; Gwak, J.; Sadeghian, A.; Reid, I.; Savarese, S. Generalized Intersection Over union: A metric and a Loss for Bounding Box Regression. In Proceedings of the IEEE Conference on Computer Vision and Pattern Recognition 2019, Long Beach, CA, USA, 15-20 June 2019; IEEE: Piscataway, NJ, USA, 2019; pp. 658-666. [CrossRef]

37. Zheng, Z.; Wang, P.; Liu, W.; Li, J.; Ye, R.; Ren, D. Distance-IoU Loss: Faster and Better Learning for Bounding Box Regression. In Proceedings of the IEEE Conference on Computer Vision and Pattern Recognition 2019, Long Beach, CA, USA, 15-20 June 2019; IEEE: Piscataway, NJ, USA, 2019. 